\title{
Note of Concern: Features of creation and use of effective heat exchangers
}

\author{
Shavkat Agzamov and Sevinar Nematova
}

Published online: 14 December 2020

Original article: E3S Web of Conferences 216 (2020),

https://doi.org/10.1051/e3sconf/202021601124

\begin{abstract}
The authors admit that they have used some data without citing the original source. They are responsible for this unintentional mistake and apologize for it. They have provided a new version with references updated.
\end{abstract}

Note of concern approved by the authors, the proceedings Editor and the Publisher on May 3, 2021. 


\title{
Features of creation and use of effective heat exchangers
}

\author{
Shavkat Agzamov ${ }^{1}$,Sevinar Nematova ${ }^{2 *}$ \\ ${ }^{1}$ Leading Specialist Uzliti Engineering, candidate of technical sciences, assistant professor. Tashkent, Uzbekistan \\ ${ }^{2}$ Assistant of the Department "Heat Power Engineering". Tashkent state technical university. Tashkent, Uzbekistan
}

\begin{abstract}
The article discusses the features of the creation and use of efficient heat exchanger. Designs of pipes with a developed heat exchange is presented. The procedure for determining the degree of development of the heat exchanging surface, the heat transfer coefficient, and the calculation of the heat transfer equation are given. As a result of creating efficient heat exchangers, three main parameters are used: the pipe outside diameter; the estimated flow rate; the Prandtl number.
\end{abstract}

\section{Introduction}

The heat exchanger will be easier to transfer heat if the heat carrier with higher values of temperature and pressure is directed inside the pipes. This allows, in the first case, to consume highly scarce high-alloy steels only for the tube sheet, and to make the casing from simpler materials. In the second case, it is easier to clean the pipes during the operation of the heat exchangers. As a rule, media for which pressure losses are limited (and these are usually gaseous media with lower pressure) are more conveniently located outside the pipes; By changing the steps of placing pipes in the bundle, the number of strokes in the annular space, it is possible to withstand the specified pressure loss.

\section{Tubular-fin heat exchanger}

If one of the coolants evaporates or condenses, then it is more convenient to send it to the annular space, otherwise, as a rule, an uneven distribution of the coolant flow through the pipes and a decrease in the efficiency of the apparatus are inevitable.

Despite the variety of heat exchangers used, it is possible to briefly formulate the basic requirements for them of a thermal, hydrodynamic, constructive, operational and technological nature: - maximum compactness, i.e. the device at the given values of thermal power and power for pumping heat carriers has a low weight and overall dimensions.

The solution to the question of which coolant should be directed inside the pipes, and which outside, depends on the pressure and temperature of the media, the convenience of the arrangement of the apparatus in the technological scheme in which it operates, on the aggressiveness of the coolant and the heat exchange surface it pollutes, on the permissible pressure losses along coolant. Of all the existing types of recuperative apparatuses, lamellar-ribbed ones have the greatest compactness, they allow placing up to $1500 \mathrm{~m}^{2}$ of heat exchange surface in $1 \mathrm{~m}^{3}$ of apparatus volume. The bodies of such devices are usually made rectangular, which excludes their use at high temperatures and pressures of coolants.

One of the varieties of tubular apparatuses are tubular-ribbed. They are used in cases where the heat transfer coefficient outside the pipes is many times less than the heat transfer coefficient inside the pipes. An increase in the heat exchange surface outside the pipes and additional turbulization of the flow by the fins can significantly increase the heat removal from the surface of the heat exchange pipes.

As previously, described in [1] improving the heathydrodynamic characteristics of heating surfaces is possible in several ways, one of which is the development of the inner and outer surfaces of pipes by radial indentation of sections of the pipe wall with the formation of depressions and protrusions of various profiles (Fig.1).

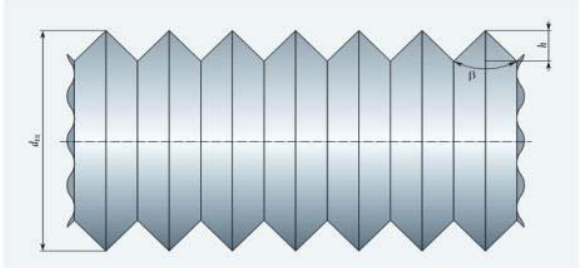

Fig.1. Scheme of the surface of the heat exchanger with triangular annular depressions and projections [1]

An increase in the surface of the heat exchanger allows one to obtain a significant development of surfaces washed by heat carriers inside and outside, as well as to intensify heat transfer due to turbulization of flows in recesses and depressions.

If we represent approximately the surface profile as a sequence of triangular protrusions and depressions and neglect the shell thickness, then a simple geometric analysis shows that the degree of 
development of the channel $\psi=\mathrm{F}_{\text {train. }} / \mathrm{F}_{\mathrm{sp}}$ depends on the angle at the apex of the triangular protrusion $\beta(0$ $<\beta<\pi$ ) and its height $\mathrm{h}$ :

$$
\Psi=\frac{F_{t r}}{F_{s p}}=\frac{1}{\sin \left(\frac{\beta}{2}\right)}\left(1-\frac{h}{d_{t r}}\right) ;
$$

where $\mathrm{F}_{\mathrm{tr}}$ - is the surface area of the pipe with triangular projections and depressions; $\mathrm{F}_{\mathrm{sp}}$ - is the surface area of a smooth pipe. The function $y=1 / \sin (\beta / 2)$ is minimal (equal to 1 ) at $\beta=\pi$ and tends to infinity as $\beta$ $\rightarrow 0$. By decreasing the angle $\beta$, one can obtain a significant increase in the heat exchange surface. So, if we take the profile of the protrusion in the form of an isosceles triangle with a right angle at the apex $\beta=$ $\pi / 2$, then the degree of surface development will be

$$
\psi=\sqrt{2}\left(1-\frac{h}{d_{s p}}\right)
$$

Hence it follows that the maximum (limiting) degree of surface development, equal to $\psi_{\lim }=\sqrt{ } 2$, is achieved at the value $\mathrm{h} \rightarrow 0$, when the number of protrusions per unit length of the pipe tends to infinity. If we take an equilateral triangle $(\beta=\pi / 3)$ as a basis, then formula (1) takes the form:

$$
\Psi=2\left(1-\frac{h}{d_{s p}}\right) \text { и } \Psi_{l i m}=2
$$

It is possible to obtain the degree of surface development $\psi>2$ by further decreasing the angle $\beta$. Then the profile of the outer and inner surfaces corresponds to the profile of the transversely finned pipe, where the conditions for heat transfer on the inner and outer surfaces are significantly deteriorated, which indicates the inexpediency of an infinite decrease in the values of the angle $\beta$. If the surface profile is represented as a sequence of semicircular protrusions and depressions (Fig. 2) and the shell thickness is neglected, it can be shown that the degree of development of the channel surface $\psi$ depends only on the diameter of the semicircular protrusion:

$$
\Psi=\frac{F_{t r}}{F_{s p}}=\frac{\pi}{2}\left(1-\frac{d}{d_{s p}}\right)
$$

In case (4), the maximum degree of surface development, equal to $\psi \lim =\pi / 2$, is reached at $d \rightarrow 0$, when the number of protrusions per unit length of the pipe tends to infinity. Any noticeable ( $\psi>1.15)$ double-sided surface development is advisable, since it directly affects the increase in the transferred heat flux and provides a corresponding decrease in the metal consumption of the heat exchanger.

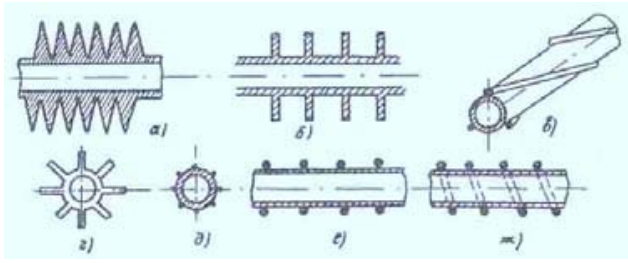

Fig. 2. Scheme of structures of heat exchange tubes with a developed heat exchange surface [1]: a - transverse helical ribbing; b - transverse annular ribs; c - spiral ribs; d longitudinal ribbing; $\mathrm{d}$ - ribbing using longitudinally welded wires; f, g - wire ribbing: circular or spiral.

\section{Diagram of heat exchange structures of pipes and elements}

The presence of finning on the outer surface of the pipes makes the pitch of the pipes in the bundle to be increased and changes the assembly technology of tubular apparatuses.

Intensification of heat transfer in pipes allows to reduce mineral deposits on the inner surface by about five times compared to smooth round pipes. In this regard, of practical interest is the class of spring-coiled channels proposed by scientists, the turns of which are made of wire of various cross-sections and rigidly fastened by laser welding. In fig. 3 shows a springtwisted channel made of round wire.

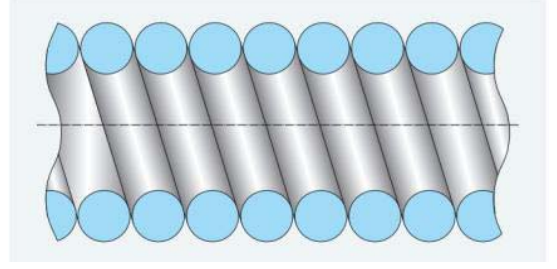

Fig. 3. Diagram of the spring-coiled channel [1]

The degree of surface development $\psi$ of springcoiled channels is determined by expressions (1) and (2). In contrast to cylindrical channels, the configuration of spring-coiled pipes provides a decrease in metal consumption compared to a smooth channel by an average of $27 \%$.

Indeed, the ratio of the mass of the material required for the manufacture of pipes of the same length is equal to the ratio of the cross-sectional areas of these channels [1].

So, for example, for a spring-twisted channel made of round wire $\frac{\mathrm{M}_{t r}}{\mathrm{M}_{c s}}$, the ratio is:

$$
\zeta=\frac{M_{t r}}{M_{c s}}=\frac{S_{t r}}{S_{c s}}=\frac{4 d^{2}}{\pi d^{2}}=\frac{4}{\pi}=1.27
$$

where $\mathrm{M}_{\mathrm{tr}}$ is the mass of a smooth pipe; $\mathrm{M}_{\mathrm{cs}}$ - mass of a spring-coiled channel made of a circular crosssection; $\mathrm{S}$ is the cross-sectional area of the wall of a smooth pipe $S_{\text {tr }}$ and a spring-twisted channel $S_{c s}$, respectively. In order to intensify heat transfer due to turbulization of the flow, it was proposed to install intensifiers 2 in the flowing part of the heat exchange element 1 , made in the form of spiral spring-wound elements rigidly fixed between the coils of a tight spring (Fig. 4).

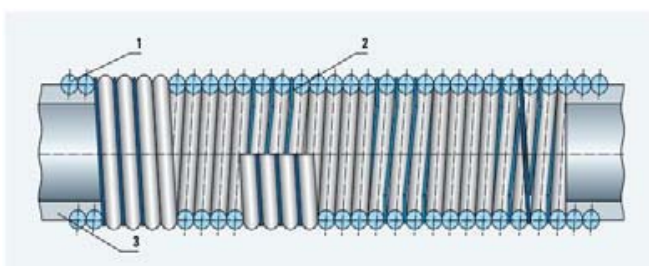

Fig. 4. Diagram of a heat exchange element with a spring intensifier [1] 
Spiral spring-wound intensifiers 2, shown in Fig. 4 , are made of elements of rectangular cross-section and, in addition, play the role of internal ribbing. The ends of such a heat exchange element are provided with branch pipes 3 for their subsequent expansion in the tube plates of shell-and-tube heat exchangers. The spring-twisted intensifiers 2 are installed by screwing them into the gaps between the turns of the stretched spring-twisted heat exchange element 1 , followed by their rigid attachment between the turns.

To increase the efficiency of heat transfer, it was also proposed to install continuous spiral intensifiers with stamped petals bent in the direction of flow swirling in the flow part of the channel. And in the work it is proposed to make the flowing part of the spring-twisted pipe in the form of projections 1 and flats 2 alternating with a given step (Fig. 5).

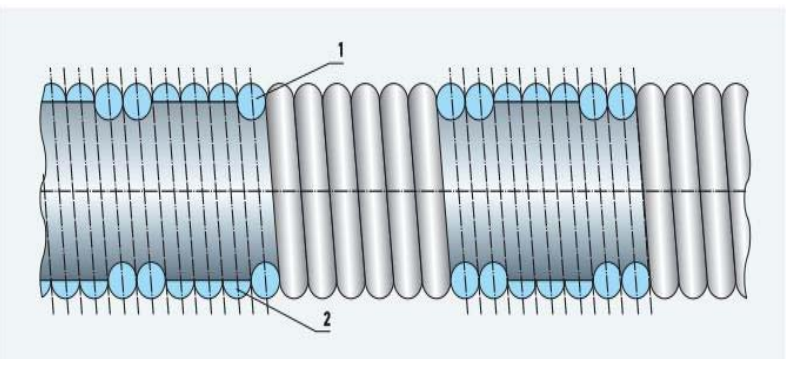

Fig. 5. Diagram of a heat exchange element with protrusions and flats [1]

The combination of zones of protrusions and flats with a predetermined step promotes rapid equalization of the temperature field in the flow path of the pipe and ensures high efficiency of heat transfer.

The design of a spring-coiled channel with converging-diffuser elements is also proposed. On the basis of the considered spring-wound channels, a series of heat exchangers is proposed. In fig. 6 shows a tube-in-tube coil heat exchanger.

The inner tube is made in the form of a springtwisted channel of circular or elliptical section, and the outer one is made of a smooth cylindrical tube. The coil heat exchanger works as follows: water is supplied to the inner pipe, and saturated steam is supplied to the annular space in a counterflow.

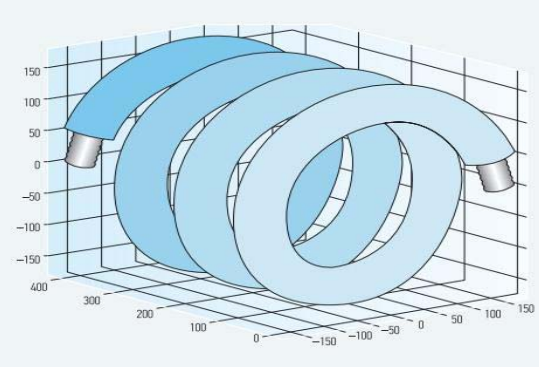

Fig. 6. Diagram of the coil spring-twisted channel [1]

With such a constructive solution, the fluid moves along a complex trajectory. Firstly, along the turns of the flowing part of the inner coil, where the swirling flow of liquid is realized along the inner depressions of the spring-coiled channel, and, secondly, along the helical line determined by the turns of the coil heat exchange element itself.

In the annular space, due to the external helical finning of the internal coil, a swirling flow with a complex three-dimensional vortex structure is realized, which intensifies the heat exchange processes occurring in the annular space. In this regard, coil heat exchangers based on spring-wound channels provide high heat transfer coefficients on the inner and outer walls of the coil elements, which can significantly increase the heat transfer coefficients and thereby reduce the overall dimensions of the apparatus in comparison with known heat exchangers of this class [1].

Thus, the proposed spring-twisted channels have a high degree of surface development, and the intensification of heat transfer in such channels makes it possible to significantly (approximately five times) reduce mineral deposits on the inner surface in comparison with smooth round pipes. The metal consumption of structures is reduced by at least $27 \%$.

In connection with the development and use of nanotechnology in the production process, it becomes possible to manufacture such pipes from non-ferrous metals (aluminum, brass, copper).

\section{Determination of the heat transfer coefficient}

For an element of the heat exchange surface $\mathrm{dF}$, the heat transfer equation in differential form is written as:

$$
\mathrm{dQ}=\mathrm{K} \Delta \mathrm{TdF}, \mathrm{Wt}
$$

where $\mathrm{K}$ - is the heat transfer coefficient, $\mathrm{W} / \mathrm{m}^{2} \mathrm{~K}$ ); $\Delta \mathrm{T}$ $=\mathrm{T}_{\mathrm{tr}}-\mathrm{T}$ - current temperature head. Total heat flux through the heat exchange surface

$$
\mathrm{Q}=\int_{0}^{F} \mathrm{~K} \Delta \mathrm{T} d F, W t
$$

To determine $\mathrm{Q}$, it is necessary to know the distribution of $\mathrm{K}$ and $\Delta \mathrm{T}$ over the heat exchange surface. For single-phase heat transfer fluids, the heat transfer coefficient usually changes insignificantly and therefore is taken constant over the entire heat exchange surface. Then

$$
\mathrm{Q}=\mathrm{K} \int_{0}^{F} \Delta \mathrm{T} d F=\kappa \overline{\Delta \mathrm{T}} F, W t
$$

where the temperature difference averaged over the heat exchange surface

$$
\overline{\Delta \mathrm{T}}=\frac{1}{\mathrm{~F}} \int_{0}^{\mathrm{F}} \Delta \mathrm{TdF},
$$

Equation (9) is the heat transfer equation. It allows to determine the heat transfer surface $\mathrm{F}$ during the design calculation. 


\section{Calculation of the heat transfer equation}

If in a heat exchanger the heat transfer coefficient significantly changes in certain areas of the heat exchange surface (as, for example, for devices with boiling or condensation of the heat carrier on a part of the surface), the coefficient $K$, averaged over the surface, is introduced [3].

For a flat wall, the heat transfer coefficient

$$
\mathrm{K}=\frac{1}{\frac{1}{\alpha_{1}}+\frac{\delta}{\lambda}+\frac{1}{\alpha_{2}}},
$$

where, $\alpha_{1}, \alpha_{2}$ - heat transfer coefficients, W / $\mathrm{m}^{2} \mathrm{~K} ; \delta$ wall thickness; $\lambda$ - coefficient of thermal conductivity of the wall material, $\mathrm{W} / \mathrm{m} * \mathrm{~K}$;

For a cylindrical wall when referring the heat flux to the inner and outer surfaces, respectively

$$
\begin{aligned}
& \mathrm{K}_{1}=\frac{1}{\frac{1}{\alpha_{1}}+\frac{d_{1}}{\lambda} \ln \frac{d_{2}}{d_{1}}+\frac{d_{1}}{\alpha_{2} d_{2}}} ; \frac{W t}{\mathrm{M}^{2} \mathrm{~K}} ; \\
& \mathrm{K}_{2}=\frac{1}{\frac{1 d_{2}}{\alpha_{1} d_{1}}+\frac{d_{2}}{2 \lambda} \ln \frac{d_{2}}{d_{1}}+\frac{1}{\alpha_{2}}} ; \quad \frac{W t}{\mathrm{M}^{2} \mathrm{~K}} ;
\end{aligned}
$$

where $\alpha_{1}, \alpha_{2}$ - the heat transfer coefficients inside and outside the pipe, $\mathrm{W} / \mathrm{m}^{2} \mathrm{~K} ; \mathrm{d} 1$ and $\mathrm{d} 2$ - inner and outer diameters.

If $\frac{d_{2}}{d_{1}}<1.8$ then it is quite acceptable to use the definition of $\mathrm{K}$ according to the formula for a flat wall (10), i.e.

$$
\mathrm{Q}=\mathrm{K} \pi d_{0} \overline{\Delta \mathrm{T}} L ; W t
$$

where $\mathrm{d}_{0}-\mathrm{d}_{2}$ at $\alpha_{1}>>\alpha_{2}, \mathrm{~d}_{0}-\mathrm{d}_{1}$ at $\alpha_{2}>>\alpha_{1}$ and $\mathrm{d}_{0}=0.5\left(\mathrm{~d}_{1}+\mathrm{d}_{2}\right)$ at $\alpha_{1} \approx \alpha_{2}$; here L- is the length of the pipes.

If we introduce a linear heat transfer coefficient for a cylindrical wall

$$
K_{l}=\frac{1}{\frac{1}{\alpha_{1} d_{1}}+\frac{1}{2 \lambda} \ln \frac{d_{2}}{d_{1}}+\frac{1}{\alpha_{2} d_{2}}} ; \quad \frac{W_{\mathrm{T}}}{\mathrm{M} \mathrm{K}} ;
$$

Then

$$
\mathrm{Q}=K_{l} \pi \overline{\Delta \mathrm{T}} L ; W t
$$

The calculated dependences necessary for determining the heat transfer coefficients are given in textbooks, monographs, reference books, for example, in [4].

In [4], recommendations are given for calculating heat transfer in pipes, annular and flat channels, longitudinally washed tube bundles.

Heat exchangers also use transversely flowed bundles of smooth tubes with a staggered and corridor arrangement. The average heat transfer for multi-row bundles of smooth pipes $(Z>10)$ is determined by the formula [4]:

$$
\mathrm{Nu}_{\mathrm{f}}=C \operatorname{Re}_{\mathrm{f}}^{\mathrm{n}} \operatorname{Pr}_{\mathrm{f}}^{0.33}\left(P r_{f} / P r_{W}\right)^{0.25} \text {, }
$$

where for in-line beams at $\operatorname{Re}_{\mathrm{f}}=$ $10^{2}, \ldots, .10^{3}$; coefficients $\mathrm{c}=0.56$ and $\mathrm{n}=0.5$. at $\mathrm{Re}_{\mathrm{f}}$ $=10^{3}-2 * 10^{5} ; \mathrm{c}=0,2 ; n=0,65=\mathrm{c}=0.2 ; \mathrm{n}=0.65$ for $\frac{\mathrm{S}_{2}}{\mathrm{D}_{\mathrm{H}}} \geq 2$ and $C=02\left[1+\left(2 \frac{\mathrm{S}_{1}}{\mathrm{D}_{\mathrm{H}}}-3\right)\left(1-\frac{\mathrm{S}_{2}}{\mathrm{D}_{\mathrm{H}}}\right)^{3}\right]^{-2}$; $n=0,65$; when $S_{2} / \mathrm{D}_{\mathrm{H}}<2 ;$; and for $S_{2} / \mathrm{D}_{\mathrm{H}} \leq 1,5$ is taken c $=0.2$; when $S_{2} / \mathrm{D}_{\mathrm{H}}>3$ is taken $S_{2} / \mathrm{D}_{\mathrm{H}}=3$.. When $\mathrm{Re}_{\mathrm{f}}>2 * 10^{5}$; the coefficients are $\mathrm{C}=0.02 ; \mathrm{p}$. $=0.84$. For chess beams $\operatorname{Re}_{\mathrm{f}}=10^{2}, \ldots, 10^{3}$; at $\mathrm{C}=$ $0.64 ; \mathrm{n}=0.5$ at $\mathrm{Re}_{\mathrm{f}}=10^{3}-2 * 10^{5}$; and

$$
\begin{gathered}
\varphi=\frac{\left(\frac{S_{1}}{D_{0}}-1\right)}{\left(\frac{s_{2}^{\prime}}{D_{0}}-1\right)}=0.1 \ldots .6 \\
S_{2}^{\prime}=\sqrt{\frac{S_{1}^{2}}{4}+S_{2}^{2}}
\end{gathered}
$$

coefficients $\mathrm{C}=0.23+0.06 \mathrm{p}=0.6$; for $\mathrm{Re}_{\mathrm{f}}>2 * 10^{5}$ coefficients $\mathrm{C}=0.023 ; \mathrm{n}=0.84$.

\section{Conclusion}

Thus, the outer pipe diameter $D_{o}$ is taken as the determining size, the average velocity in a narrow cross-section of the beam is taken as the design flow rate, the average flow temperature is taken as the determining temperature $\mathrm{T}_{f}$, and the Prandtl number $\mathrm{P}_{r w}$ is determined by the average wall temperature. For gases $\left(\frac{P r_{f}}{P r_{W}}\right)=1$.

\section{References}

1. A.G. Bagougdinova, Ya. D. Zolotonosov, V.N. Posokhin. Design of effective heat exchange elements for high-speed heat exchangers / Plumbing. Heating. Conditioning. 2014. No. 7. - P.72-75.

2. Pismenniy E.N., Baranyuk M.M., Voznyuk M.M. Raznorazvitiye povexnosti teploobmena I metodika chislennix ix teplogidravlicheskix xarakteristik //Promishlennaya teplotexnika, / 2012. №1. 13-18 p.

3. Dzyubenko B.N. Vliyanie zakrutki potoka na teplomassoobmen $\mathrm{v}$ usloviyax soleotlojeniy $\mathrm{v}$ vitix trubax // Trudi IV-y Ros. nats. konf. Po teploobm.:T.6. Dispersnie potoki I poristie sredi. Intensifikatsiya teploobmena. -M.: Izd-vo MEI, 2006.- 312 p.

4. Kalinin E.K., Dreytser G.A., Yarxo S.A. Intensifikatsiya teploobmena $\mathrm{v}$ kanalax. - M.: Mashinastroenie, 1981. - $205 \mathrm{p}$.

5. Uspexi teploperedachi. T.2 Intensifikatsiya teploobmena / pod. Red. E.K.Kalinina // Vilnyus, «Мокслас», 1988 г.-187 p. 